\title{
Lipoprotein-Associated Phospholipase A2 Activity and Mass as Independent Risk Factor of Stroke: A Meta-Analysis
}

\author{
Gaifeng Hu, ${ }^{1,2}$ Deping Liu, ${ }^{2}$ Huiyu Tong, ${ }^{1}$ Weijun Huang, \\ Yunzhao Hu ${ }^{D}{ }^{1}$ and Yuli Huang ${ }^{1}{ }^{1}$ \\ ${ }^{1}$ Department of Cardiology, Shunde Hospital of Southern Medical University, Foshan 528300, China \\ ${ }^{2}$ Department of Cardiology, Beijing Hospital, National Center of Gerontology, No.1 DaHua Road, Dong Dan, \\ Graduate School of Peking Union Medical College, Beijing, 100730, China \\ Correspondence should be addressed to Yunzhao Hu; huyunzhao4406@163.com and Yuli Huang; hyuli821@163.com
}

Received 7 March 2019; Accepted 24 April 2019; Published 20 May 2019

Academic Editor: Vida Demarin

Copyright (C) 2019 Gaifeng Hu et al. This is an open access article distributed under the Creative Commons Attribution License, which permits unrestricted use, distribution, and reproduction in any medium, provided the original work is properly cited.

\begin{abstract}
Background. The association between lipoprotein-associated phospholipase A2 (Lp-PLA2) and stroke risk is inconsistent. We conducted a meta-analysis to determine whether elevated Lp-PLA2 is a risk factor for stroke. Methods. Studies were included if they reported Lp-PLA2 mass and/or activity levels and adjusted risk estimates of stroke. The primary outcome was overall stroke incidence. The combined results were shown as relative risks (RRs) with 95\% confidence intervals (CI) for per 1 standard deviation (SD) higher value of Lp-PLA2 and the highest versus lowest Lp-PLA2 category. Results. Twenty-two studies involving 157,693 participants were included for analysis. After adjusting for conventional risk factors, the RRs for overall stroke with 1 SD higher Lp-PLA2 activity and mass were 1.07 (95\% CI 1.02-1.13) and 1.11 (95\% CI 1.04-1.19), respectively. The RRs of ischemic stroke with 1 SD higher Lp-PLA2 activity and mass were 1.08 (95\% CI 1.01-1.15) and 1.11 (95\% CI 1.02-1.22), respectively. When comparing the highest and lowest levels of Lp-PLA2, the RRs of stroke for Lp-PLA2 activity and mass were 1.26 (95\% CI 1.03-1.54) and 1.56 (95\% CI 1.21-2.00), respectively. Finally, when comparing the highest and lowest levels of Lp-PLA2, the pooled RRs of ischemic stroke for Lp-PLA2 activity and mass were 1.29 (95\% CI 1.07-1.56) and 1.68 (95\% CI 1.12-2.53), respectively. Conclusions. Elevated baseline Lp-PLA2 levels, detected either by activity or mass, are associated with increased stroke risk.
\end{abstract}

\section{Introduction}

Stroke results in $9 \%$ of all deaths and is the primary cause of disability around the world [1]. Tailored treatment based on risk factors may help to target therapies to high-risk patients and reduce stroke morbidity. Biomarkers are being investigated to improve stroke diagnosis and determine its cause.

Lipoprotein-associated phospholipase A2 (Lp-PLA2) is an enzyme mainly produced by macrophages and monocytes [2] and can hydrolyze oxidized phospholipids, produce oxidative modification of low-density lipoprotein, and release pro-inflammatory and pro-atherogenic metabolites. Thus, Lp-PLA2 may contribute to the development of atherosclerosis and plaque rupture, which in turn leads to coronary heart disease and stroke [3].
Although the US Food and Drug Administration approved a Lp-PLA2 blood test for assessing patients at risk for ischemic stroke in 2005, research into the association between Lp-PLA2 and stroke risk has yielded inconsistent results. The association of circulating Lp-PLA2 with ischemic stroke is less clear than with coronary heart disease, perhaps because fewer outcomes in ischemic stroke are recorded. Levels of Lp-PLA2 are measured in two ways: Lp-PLA2 activity and mass assays. Although Lp-PLA2 activity correlates with Lp-PLA2 mass [4], the risk of ischemic stroke with Lp-PLA2 activity and mass is inconsistent; for example, a meta-analysis of individual patient data (IPD) demonstrated that higher Lp-PLA2 mass, but not activity, may increase ischemic stroke risk [5]. More recently, however, increasing numbers of studies have reported an association between Lp-PLA2 and stroke risk. We conducted 
a meta-analysis to evaluate the association between baseline levels of Lp-PLA2 activity/mass and stroke risk.

\section{Methods}

2.1. Literature Search. This study was conducted according to the Preferred Reporting Items for Systematic Reviews and Meta-Analyses (PRISMA) statement [6] (Supplement 1). We searched relevant studies in PubMed, Embase, and Cochrane Library to February 2019. There were no restrictions on language or the publication date. Relevant studies were identified using following terms: "Lipoprotein-Associated Phospholipase A2" OR "1-Alkyl-2-acetylglycerophosphocholine Esterase” OR “Lp-PLA2" AND “stroke” OR “cardiovascular disease" OR "cerebrovascular disorder" OR "cardiocerebrovascular disease" OR "cardiovascular event" OR "cerebrovascular disease" OR "cerebrovascular attack" OR "cerebral infarction" OR "intracranial hemorrhage" OR "intracerebral hemorrhage" OR "Transient Ischemic Attack". The detailed search strategy is presented in Online Supplement 2.

2.2. Study Selection Criteria. Studies were included if (1) they were prospective cohort studies (reported as observational cohort studies or case-cohort subsets) or randomized controlled trials with data on Lp-PLA2 mass and/or activity at baseline; (2) multivariate-adjusted relative risk ratios (RR) of stroke or transient ischemic attack (TIA) associated with LpPLA2 mass and/or activity were reported; and (3) participants were aged $\geq 18$ years.

Studies were excluded if (1) they were retrospective or cross-sectional studies, (2) RR was adjusted for age and sex only, (3) RR with 95\% confidence intervals (95\% CI) were reported from only two quantitative exposure categories, or (4) data were derived from the same cohort or from combined analysis of other studies.

2.3. Data Extraction and Quality Assessment. Two authors (Gaifeng Hu and Deping Liu) extracted relevant information from the eligible studies independently. Any divergences were resolved by discussion. Study quality was independently assessed by two authors (Huiyu Tong and Weijun Huang) conforming to the Newcastle Ottawa Scale (NOS) [7]. Studies getting a rating of $\geq 8$ stars on the NOS indicated the high study quality; all others were considered as low or moderate quality.

2.4. Pooled Data Analysis. The primary outcome was stroke, including ischemic stroke, hemorrhagic stroke, unclassified stroke, and TIA. The secondary outcome was ischemic stroke. Subgroup analyses of the primary outcome were conducted according to sex, age, study design, follow-up period, inclusion of participants with baseline cardiovascular disease (CVD; yes vs. no), inclusion of participants with baseline CVD type, ischemic stroke event sizes, and NOS quality.

Multivariate-adjusted RR or hazard ratios with 95\% CI were used for analysis. The inverse variance approach was used to combine the log RR and SE values.
We performed two comparisons. First, we compared stroke risk in individuals with the highest quantile of LpPLA2 with those with the lowest quantile of Lp-PLA2. Second, we calculated the RR for a 1-standard-deviation (SD) change in baseline levels of Lp-PLA2 mass and activity.

We used $\chi^{2}$ and $\mathrm{I}^{2}$ statistics to examine heterogeneity. Values of $P<0.10$ or $\mathrm{I}^{2}>50 \%$ indicated significant heterogeneity across studies. We also reported results from random-effects models. Publication bias was assessed by Begg's test, and a sensitivity analysis was conducted by omitting one study at a time.

Statistical analyses were conducted using RevMan software (v5.3 for Windows, The Cochrane Collaboration, Copenhagen, Denmark) and Stata (v13.0, Stata Corp LP, College Station, Texas). A value of $P<0.05$ was considered statistically significant.

\section{Results}

3.1. Study Characteristics. From the initial search, 718 publications were identified. After removing 193 duplications, we reviewed titles and abstracts and excluded 399 manuscripts. We then thoroughly assessed the full text from 126 papers. Finally, 22 publications [4, 5, 8-27] were included in our analyses (Figure 1).

The key features of included studies are reported in Table 1; there were 22 studies with 157,693 participants. Among them, the Cardiovascular Health Study (CHS) was separated into two studies by race (CHS-1: whites and CHS2: African Americans). The Heart Protection Study (HPS) was stratified by stroke subtype (HPS-1: ischemic stroke and HPS-2: hemorrhagic stroke). The results of methodological quality for each included study are presented in Supplement 3. Overall, study quality was of moderate to high quality (ranging from 5-9).

3.2. Association of Lp-PLA2 Activity and Stroke Risk. Seventeen studies reported RR for total stroke with 1 SD higher LpPLA2 activity, and the pooled adjusted RR was 1.07 (95\% CI $1.02-1.13 ; P=0.008$ ) in a random-effects model (Figure 2(a)). Similarly, pooled data from nine studies demonstrated that the risk of ischemic stroke with 1 SD higher Lp-PLA2 activity was 1.08 (95\% CI 1.01-1.15; $P=0.02$; Figure 2(b)).

From 11 studies, the pooled adjusted RR of all stroke when comparing the highest with the lowest Lp-PLA2 activity was 1.26 (95\% CI 1.03-1.54; $P=0.008)$ in a random-effects model (Figure 3(a)). Furthermore, the pooled adjusted RR of ischemic stroke comparing the highest with the lowest Lp-PLA2 activity was 1.29 (95\% CI 1.07-1.56; $P=0.009$; Figure 3(b)).

3.3. Association of Lp-PLA2 Mass and Stroke Risk. Data from 10 studies demonstrated that the risk of all stroke when comparing the highest with the lowest Lp-PLA2 mass was 1.56 (95\% CI 1.21-2.00; $P=0.0006)$ in a random-effects model (Figure 4(a)). Furthermore, the pooled RR of ischemic stroke comparing the highest with the lowest Lp-PLA2 mass was 1.68 (95\% CI 1.12-2.53; $P=0.01$; Figure 4(b)). 


\begin{tabular}{|c|c|c|c|c|c|c|c|c|}
\hline 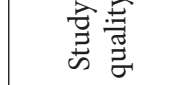 & $a$ & $a$ & $a$ & $a$ & 6 & $\infty$ & $a$ & $a$ \\
\hline 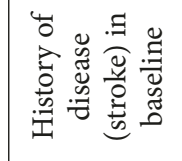 & \multicolumn{4}{|c|}{ 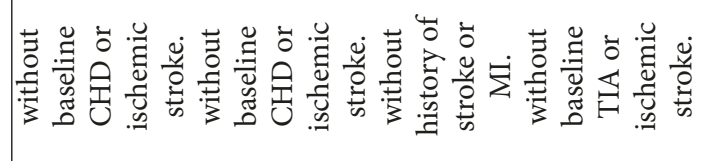 } & \multicolumn{4}{|c|}{ 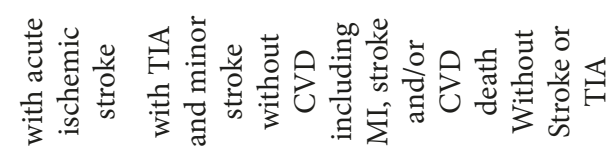 } \\
\hline 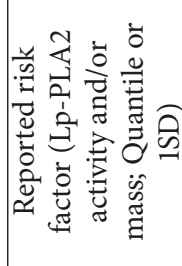 & 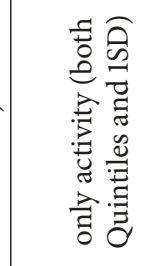 & 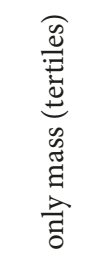 & 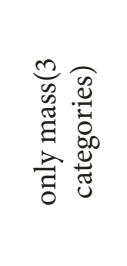 & 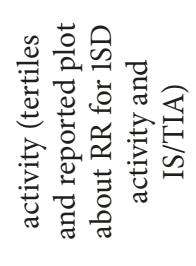 & 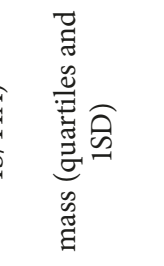 & 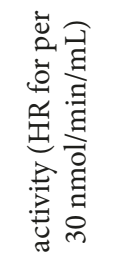 & 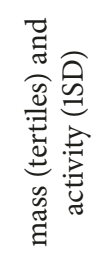 & 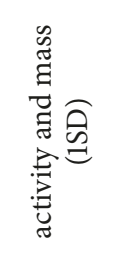 \\
\hline 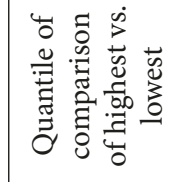 & 它竞 & $\underset{\substack{0 \\
\vec{D}}}{\stackrel{0}{ \pm}}$ & 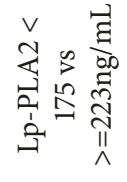 & 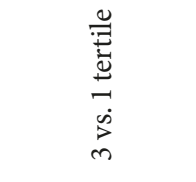 & 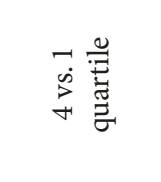 & & 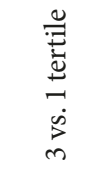 & ' \\
\hline 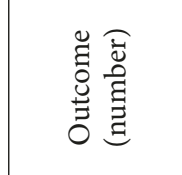 & 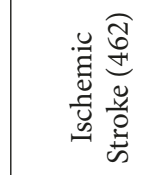 & 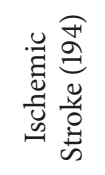 & 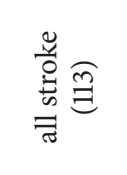 & 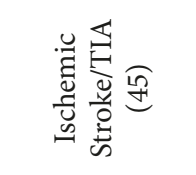 & 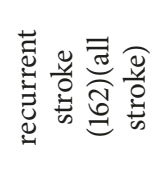 & 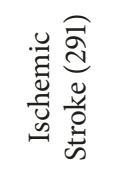 & 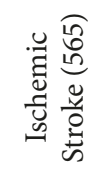 & 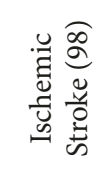 \\
\hline 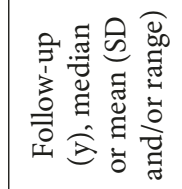 & 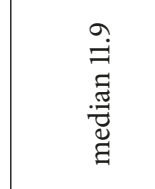 & 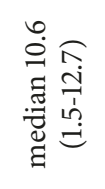 & 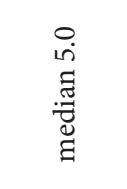 & 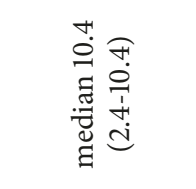 & - & 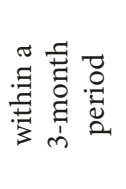 & $\begin{array}{l}\stackrel{n}{\exists} \\
\underset{\Xi}{\Xi} \\
\stackrel{\Xi}{\Xi}\end{array}$ & $\begin{array}{l}\stackrel{\infty}{a} \widehat{\overparen{\Xi}} \\
\stackrel{\Xi}{\Xi}\end{array}$ \\
\hline 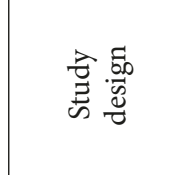 & 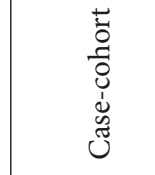 & 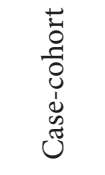 & 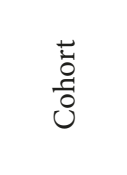 & $\begin{array}{l}\frac{\pi}{0} \\
\frac{1}{0} \\
0\end{array}$ & 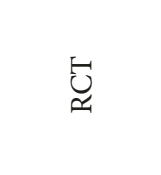 & $\ddot{\mathscr{u}}$ & $\begin{array}{l}\overline{0} \\
\overline{0} \\
0\end{array}$ & 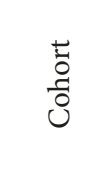 \\
\hline 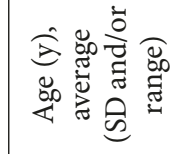 & 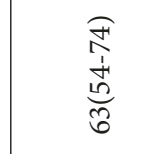 & $\underset{\text { in }}{\stackrel{1}{f}} \underset{6}{F}$ & 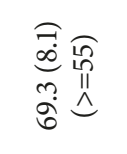 & $\hat{\widehat{\sigma}}$ & 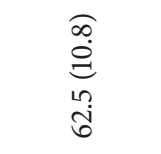 & 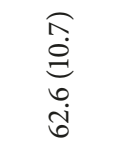 & $\frac{\sqrt{0}}{\infty}$ & $\begin{array}{l}\hat{\sigma} \\
\underline{0} \\
\dot{0}\end{array}$ \\
\hline 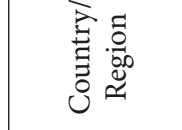 & 崩 & 崩 & 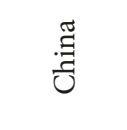 & 氞 & 节 & 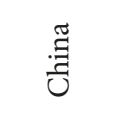 & 岕 & 峁 \\
\hline 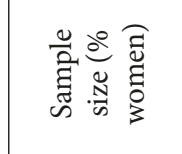 & $\begin{array}{l}0 \\
\stackrel{0}{0} \\
N \\
\cong\end{array}$ & $\begin{array}{l}\text { on } \\
\stackrel{+}{+1} \\
\text { 点 } \\
0 \\
0\end{array}$ & 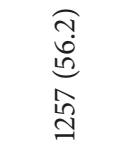 & 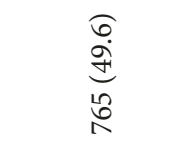 & 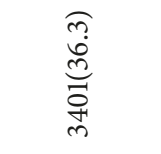 & $\begin{array}{l}\widehat{\infty} \\
\stackrel{0}{0} \\
\vec{c} \\
\vec{\delta}\end{array}$ & 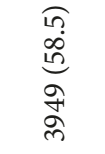 & 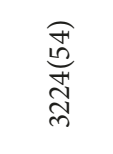 \\
\hline 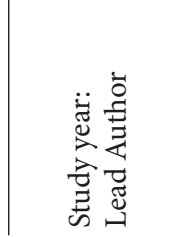 & 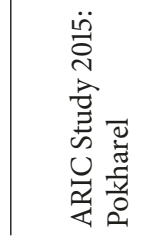 & 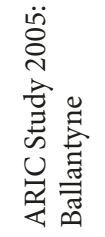 & 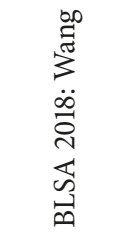 & 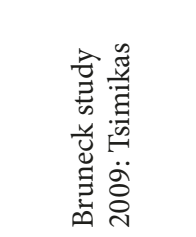 & 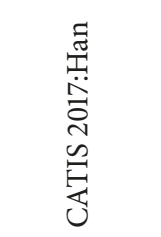 & 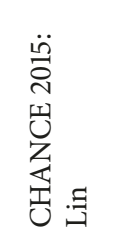 & 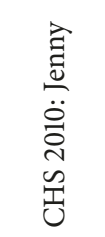 & 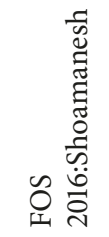 \\
\hline
\end{tabular}




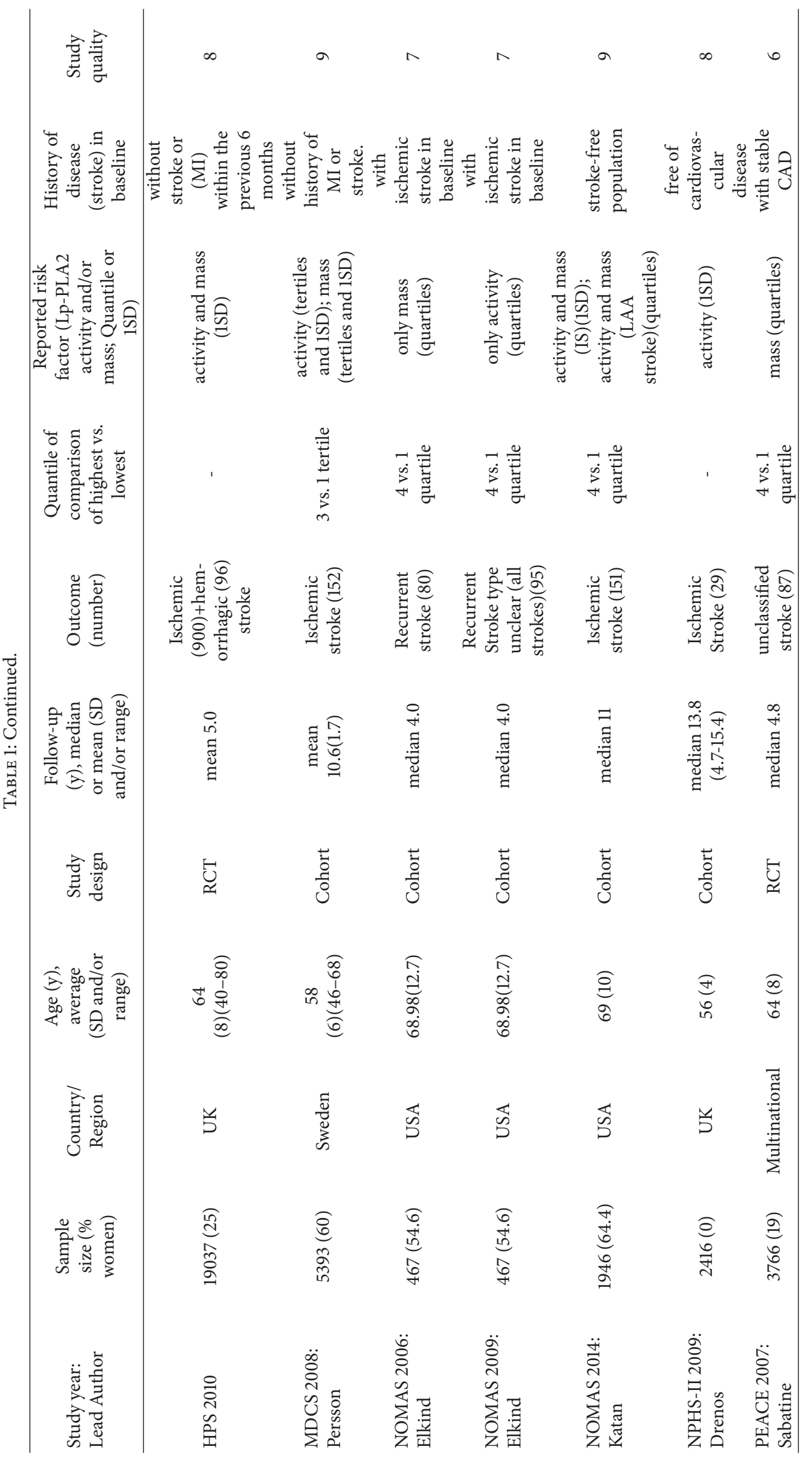




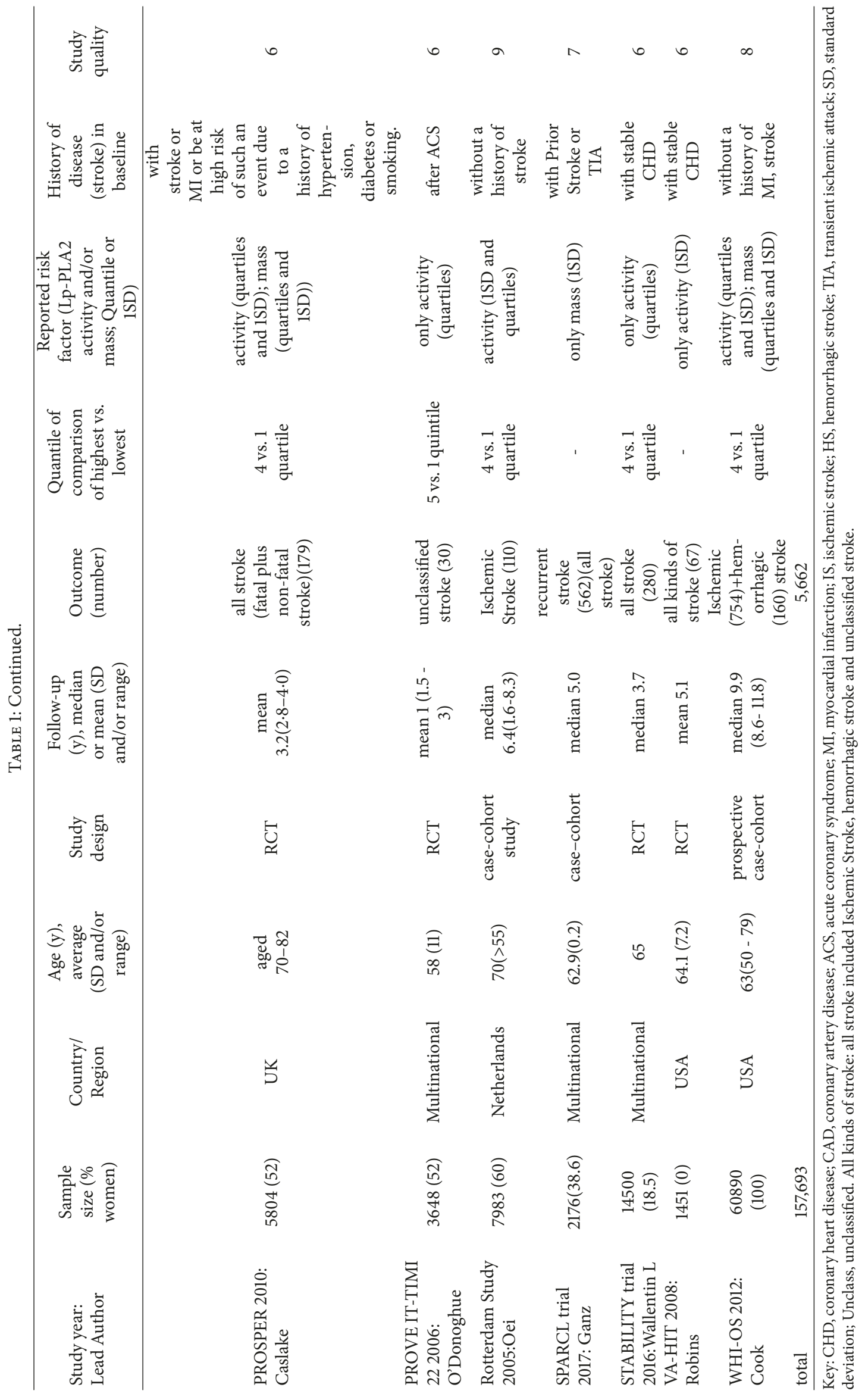




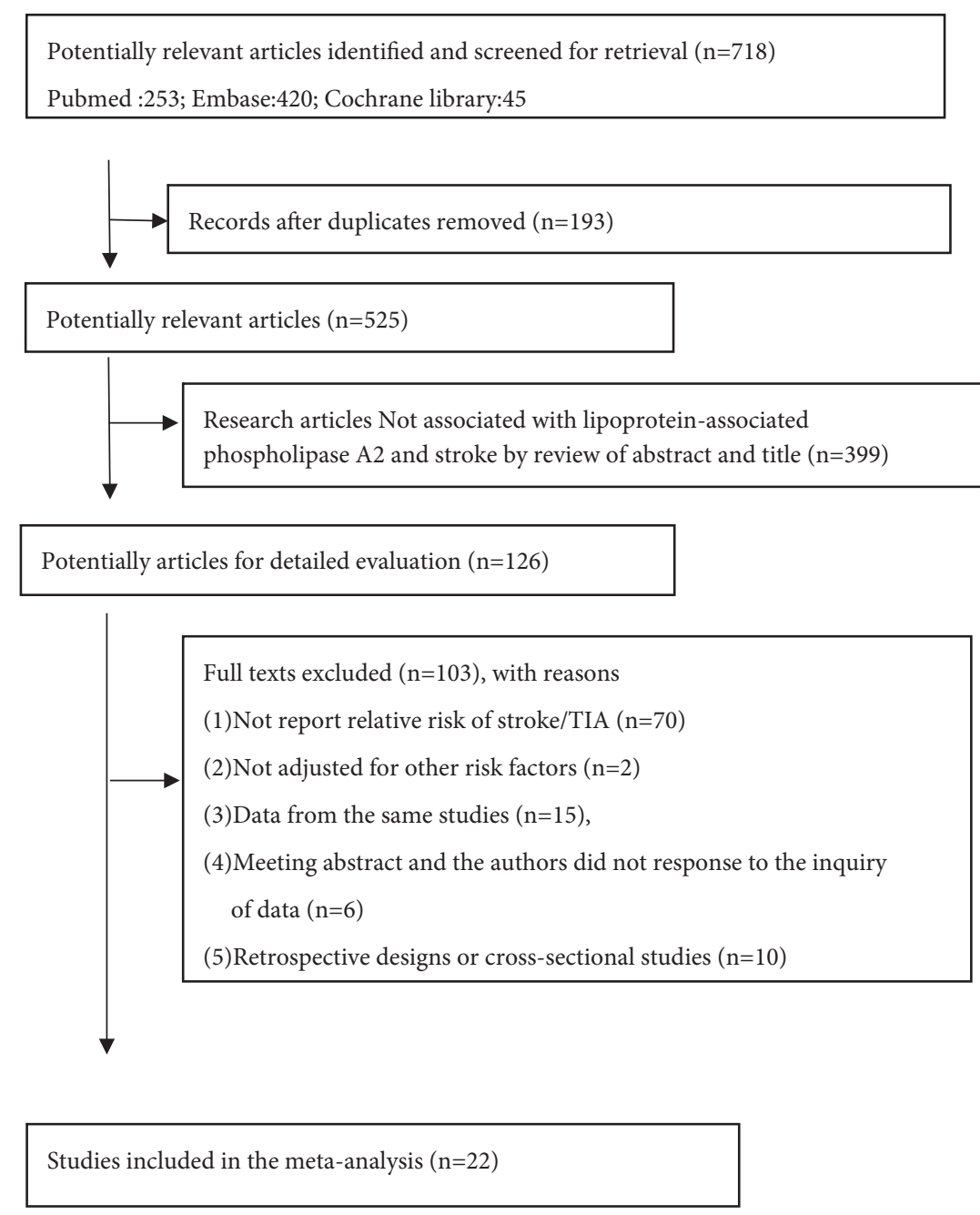

Figure 1: Flow chart of study selection.

Thirteen studies reported total stroke risk with 1 SD higher Lp-PLA2 mass. The pooled RR from these studies was 1.11 (95\% CI 1.04-1.19; $P=0.003$; Figure 5(a)). In addition, meta-analysis of seven studies demonstrated that ischemic stroke risk increased per $1 \mathrm{SD}$ increase of Lp-PLA2 mass (RR, 1.11; 95\% CI 1.02-1.22; $P=0.02$; Figure 5(b)).

3.4. Publication Bias and Sensitivity Analyses. There was no evidence of publication bias in any analysis, as indicated by Begg's rank correlation test (all $P>0.1$ ). Sensitivity analyses demonstrated limited influence in the quantitative pooled RR and its 95\% CI for total stroke with 1 SD higher Lp-PLA2 activity and mass. When any individual study was omitted, results remained consistent, suggesting that our conclusion was reliable.

3.5. Subgroup Analyses Based on per SD Change of LP-PLA2 Activity. Elevated Lp-PLA2 activity (per $1 \mathrm{SD}$ ) and greater stroke risk were observed consistently in subgroups, except for the subgroups defined as with a prospective cohort study design (RR 1.08; 95\% CI 0.99-1.17), mean age $\geq 65$ years, follow-up period $\geq 10$ years, history of CVD (including coronary heart disease (CHD), stroke and TIA), NOS quality scores $<8$ stars, or when men and women were analyzed separately (Supplement 6,7), which may be caused by inadequate numbers and low statistical power. However, none of the differences between these subgroups were significant (all $P>0.1)$.

\section{Discussion}

In this meta-analysis, after adjusting for multiple conventional risk factors, elevated Lp-PLA2 levels of both activity and mass were associated with increased risk of stroke, including ischemic stroke. Per 1 SD higher value of LpPLA2 activity and mass, there was $7 \%$ and $11 \%$ greater risk of all stroke, respectively, and $8 \%$ and $11 \%$ greater risk of ischemic stroke, respectively. Subjects with the highest LpPLA2 activity and mass had respective $26 \%$ and $56 \%$ greater risk of all stroke compared with those with the lowest activity and mass. 


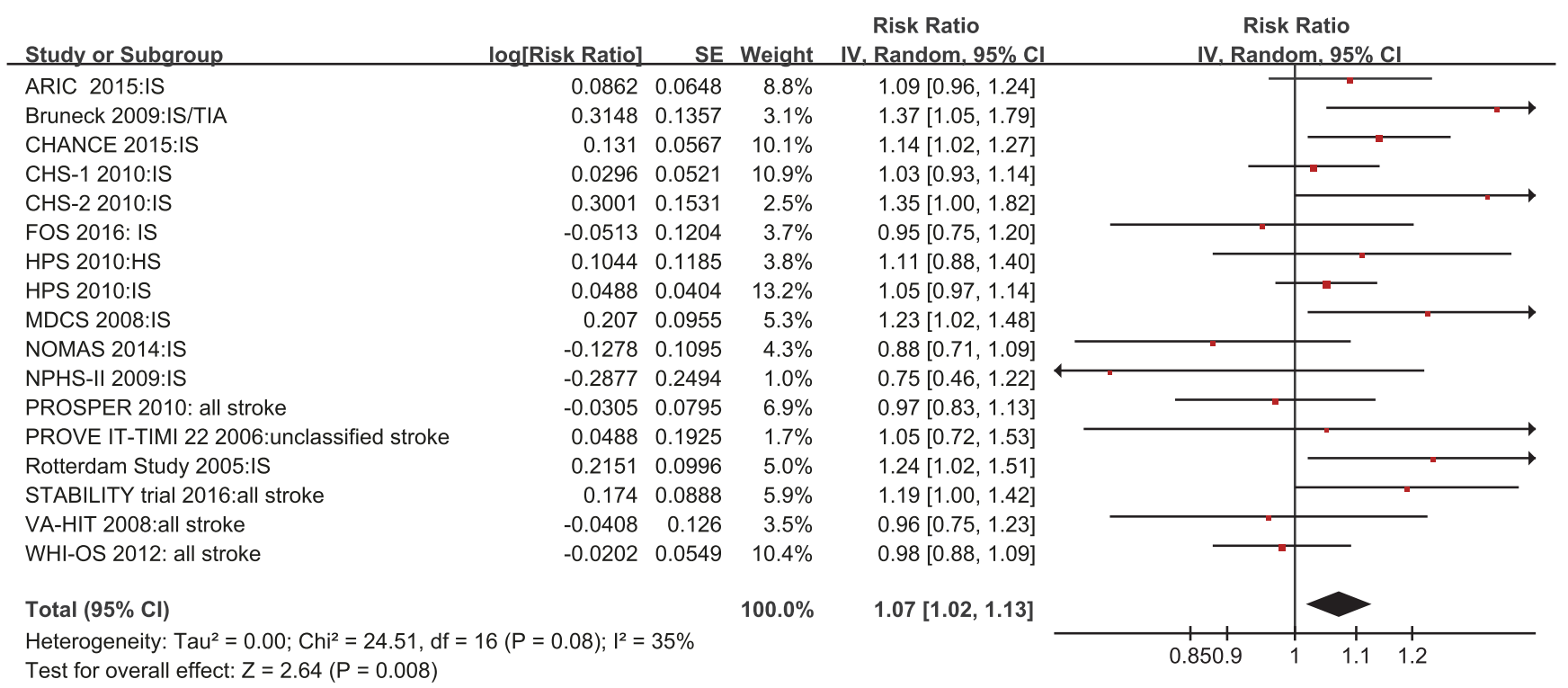

(a)

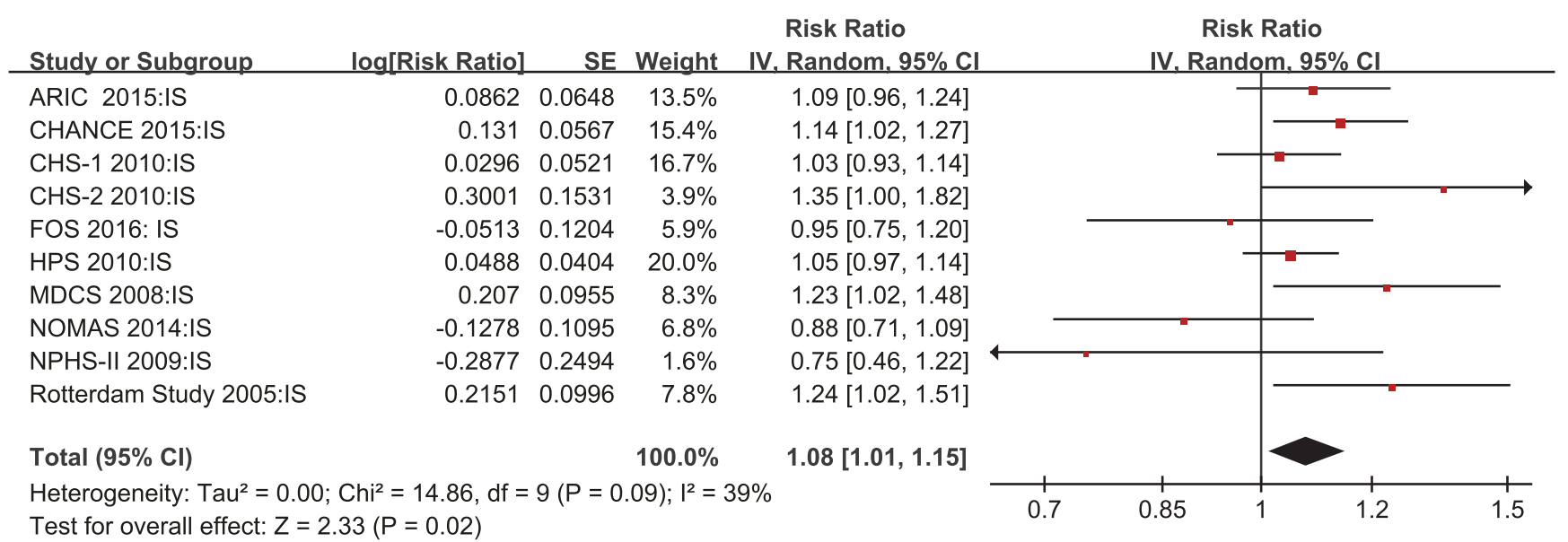

(b)

FIGURE 2: Forest plot of pooled RRs for all stroke (a) and ischemic stroke (b) with 1 SD higher Lp-PLA2 activity.

A previous meta-analysis used IPD, and the prognostic strength of Lp-PLA2 for coronary heart disease was determined by a continuous variable analysis [5]. This IPD meta-analysis found that, per $1 \mathrm{SD}$ increase in Lp-PLA 2 mass, there was increased ischemic stroke risk (RR 1.14; 95\% CI 1.02-1.27), and per 1 SD increase in LpPLA2 activity, there was significantly increased CHD risk (RR 1.10, 95\% CI 1.05-1.16). However, a 1 SD increase in Lp-PLA2 activity was not associated with ischemic stroke risk (RR 1.08; 95\% CI 0.97-1.20).

There are two methods to test Lp-PLA2 in human plasma and serum including its plasma concentration (mass) and enzymatic activity. However, Lp-PLA2 mass measurement has been gradually replaced, as it is less accurate than enzymatic activity assessment for risk stratification [28]. The methods for the detection of Lp-PLA2 activity in human blood have been developed and all resulted in more accurate than mass measurement in testing the total levels of circulating Lp-PLA2 [28]. In 2014, The FDA approved a LpPLA2 activity test by use of the PLAC ${ }^{\circledR}$ Test (diaDexus Inc, San Francisco, CA, USA) to predict cardiovascular disease in clinical practice.

In our study, elevated Lp-PLA2 activity was related to higher ischemic stroke risk (RR 1.08; 95\% CI 1.01-1.15), possibly because we included newer and more relevant studies to increase the statistical power, particularly in the subgroup with mean age $<65$ years. There was also an increased trend of all stroke and ischemic stroke risk in subjects with mean age $\geq 65$ years with 1-SD-elevated Lp-PLA2 activity levels, although this was not statistically significant. These findings suggest that the relationship between Lp-PLA2 activity levels and ischemic stroke in individuals $<65$ years is more pronounced than that in individuals $\geq 65$ years. However, this may be because of lack of studies in individuals $\geq 65$ years, which leads to low statistical power. Further research into the association between LPPLA2 activity and 


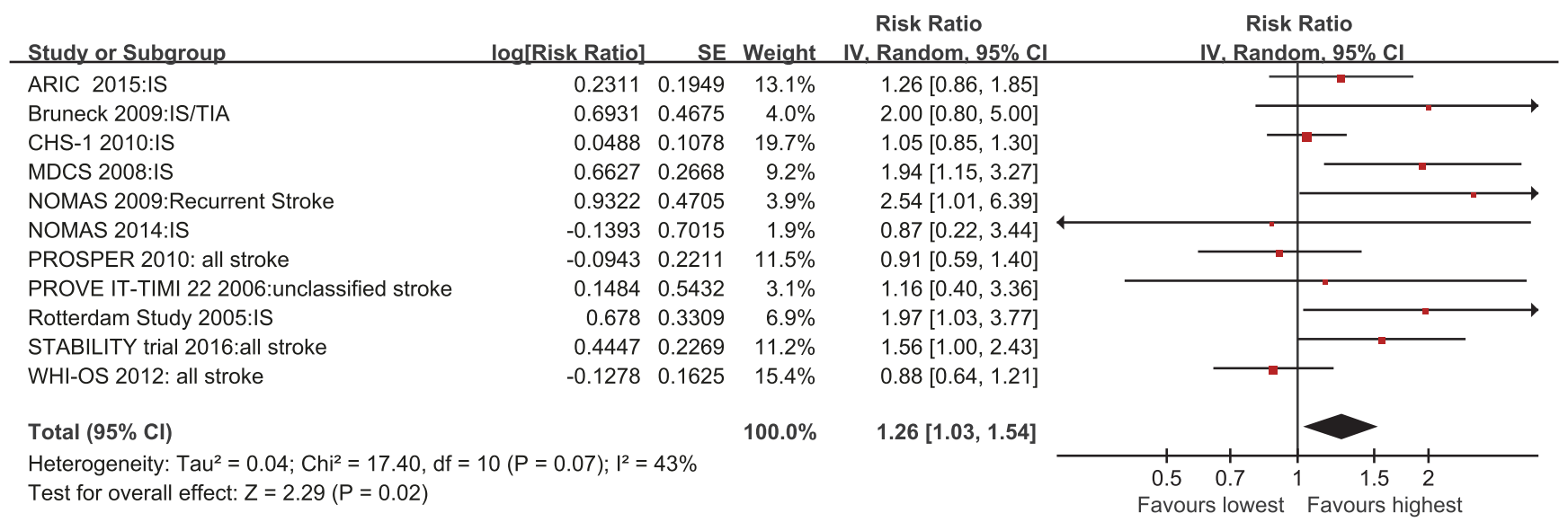

(a)

Risk Ratio Risk Ratio

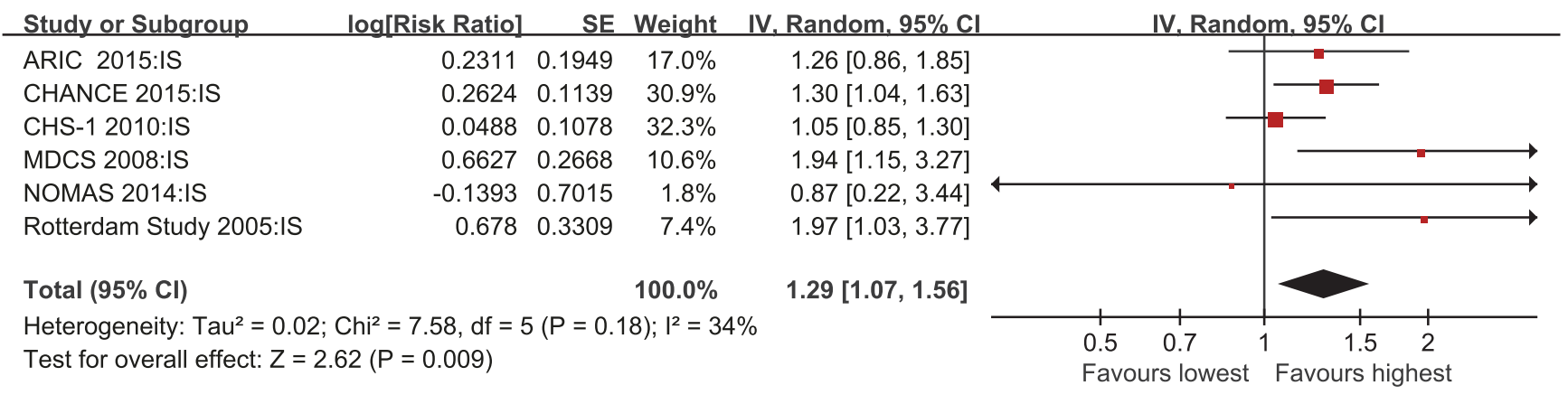

(b)

FIGURE 3: Forest plot of pooled RRs of all stroke (a) and ischemic stroke (b) for Lp-PLA2 activity comparing the highest with the lowest levels of Lp-PLA2.

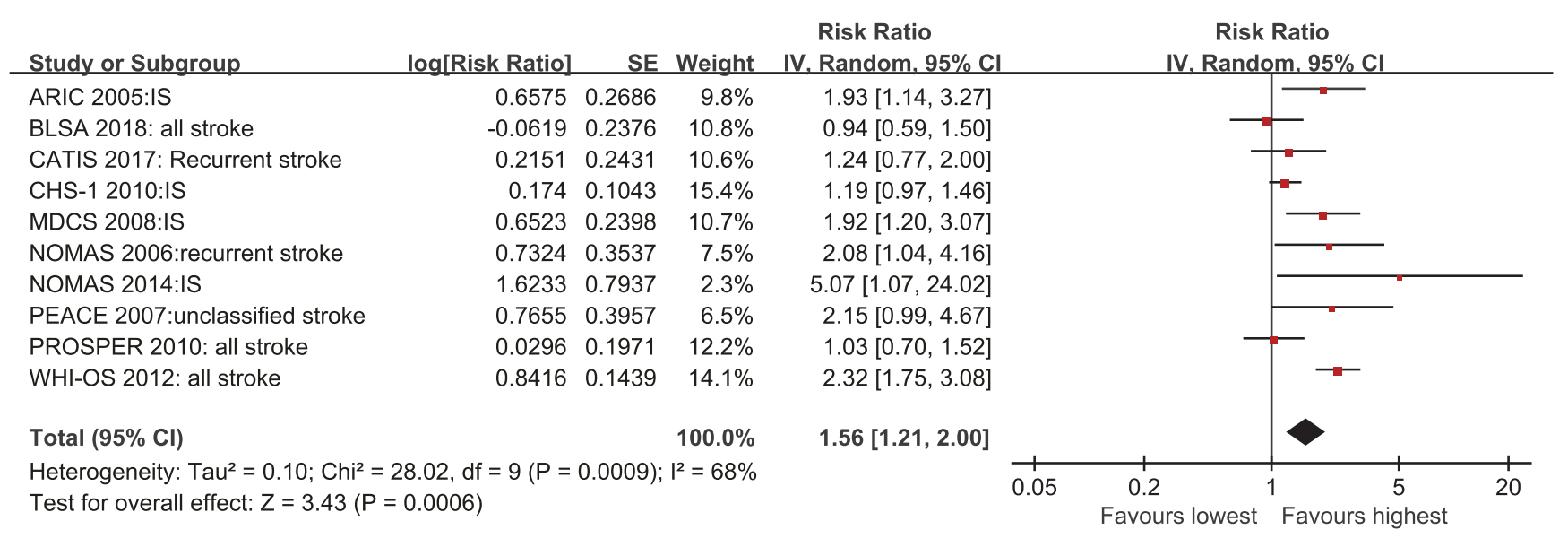

(a)

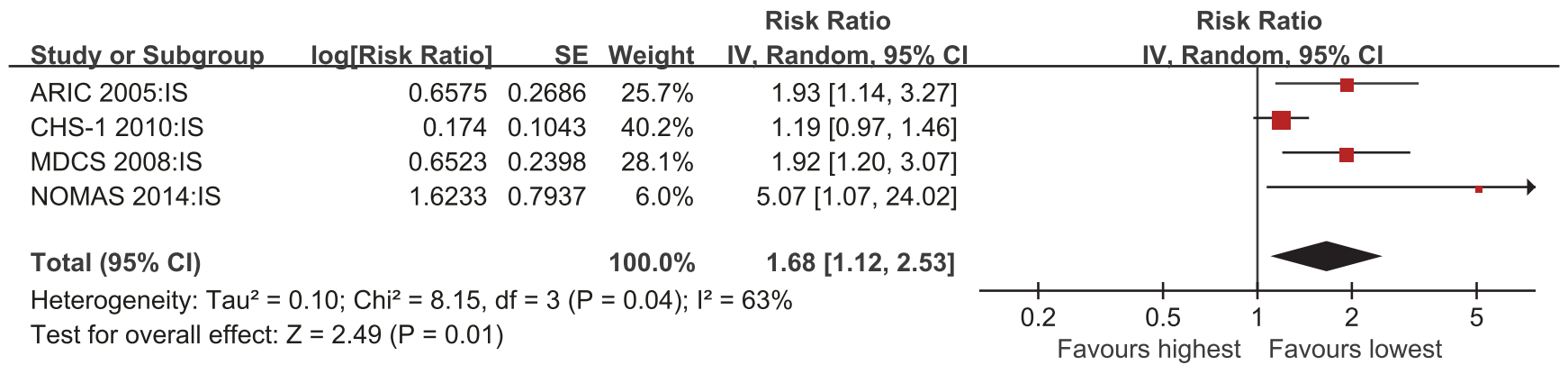

(b)

FIGURE 4: Forest plot of pooled RRs of all stroke (a) and ischemic stroke (b) for Lp-PLA2 mass comparing the highest with the lowest levels of Lp-PLA2. 


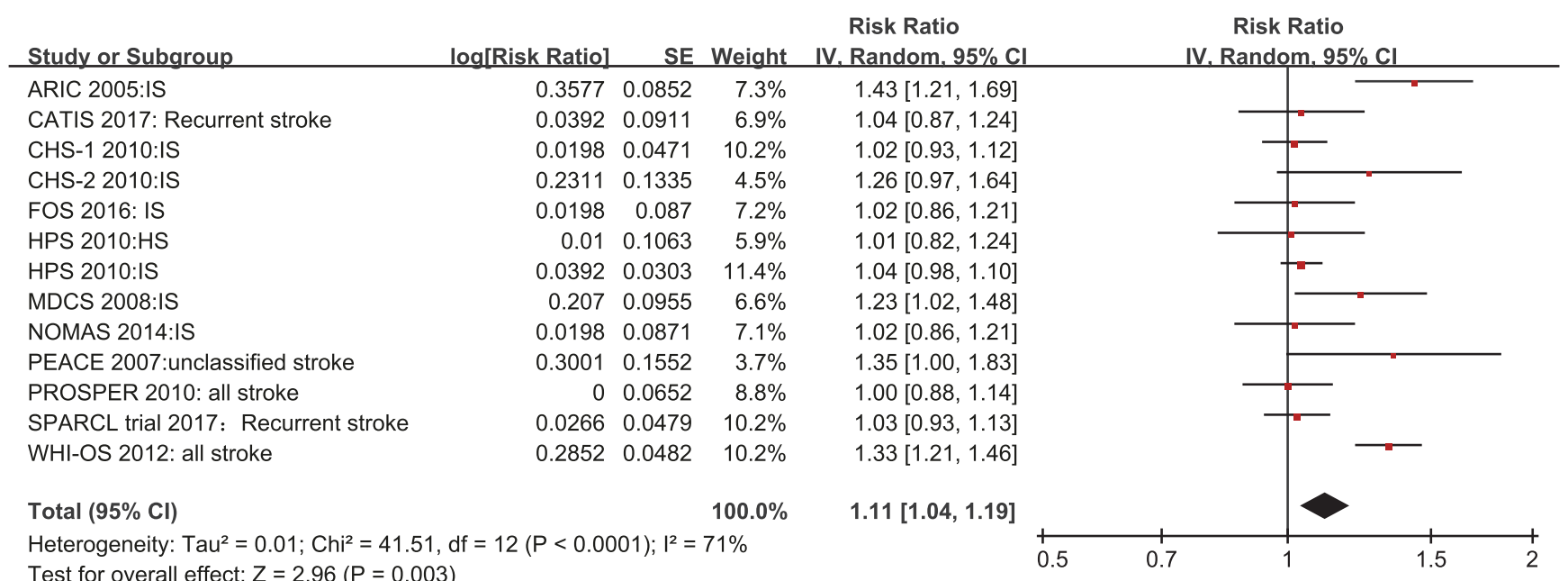

(a)

\begin{tabular}{|c|c|c|c|c|c|c|c|c|}
\hline Study or Subgroup & log[Risk Ratio] & SE & Weight & $\begin{array}{l}\text { Risk Ratio } \\
\text { IV, Random, } 95 \% \mathrm{Cl}\end{array}$ & & IV, $\mathbf{F}$ & $\begin{array}{l}\text { Ratio } \\
\text { om, } 95 \% \mathrm{Cl}\end{array}$ & \\
\hline ARIC 2005:IS & 0.3577 & 0.0852 & $13.1 \%$ & $1.43[1.21,1.69]$ & & & & \\
\hline CHS-1 2010:IS & 0.0198 & 0.0471 & $19.4 \%$ & $1.02[0.93,1.12]$ & & & 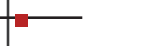 & \\
\hline CHS-2 2010:IS & 0.2311 & 0.1335 & $7.8 \%$ & $1.26[0.97,1.64]$ & & & & \\
\hline FOS 2016: IS & 0.0198 & 0.087 & $12.9 \%$ & $1.02[0.86,1.21]$ & & & & \\
\hline HPS 2010:IS & 0.0392 & 0.0303 & $22.2 \%$ & $1.04[0.98,1.10]$ & & & & \\
\hline MDCS 2008:IS & 0.207 & 0.0955 & $11.7 \%$ & $1.23[1.02,1.48]$ & & & & \\
\hline NOMAS 2014:IS & 0.0198 & 0.0871 & $12.9 \%$ & $1.02[0.86,1.21]$ & & & & \\
\hline Total $(95 \% \mathrm{Cl})$ & & & $100.0 \%$ & $1.11[1.02,1.22]$ & & & & \\
\hline \multicolumn{5}{|c|}{$\begin{array}{l}\text { Heterogeneity: } \mathrm{Tau}^{2}=0.01 ; \mathrm{Chi}^{2}=17.74, \mathrm{df}=6(P=0.007) ; \mathrm{I}^{2}=66 \% \\
\text { Test for overall effect: } Z=2.37(P=0.02)\end{array}$} & 0.7 & 0.85 & 1.2 & 1.5 \\
\hline
\end{tabular}

(b)

FIGURE 5: Forest plot of pooled RRs for all stroke (a) and ischemic stroke (b) with 1 SD higher Lp-PLA2 mass.

ischemic stroke in individuals $\geq 65$ years is thus needed to confirm this relationship. Patients with 1 SD higher Lp-PLA2 activity had a $16 \%$ increased risk of recurrence after cerebral vascular diseases including ischemic stroke, minor stroke, and TIA, demonstrating powerful prognostic strength of LpPLA2 activity for stroke in individuals with baseline ischemic stroke, minor stroke, or TIA. However, there was no increased stroke risk in participants with baseline CHD (RR 1.04; 95\% CI 0.94-1.15; supplement 7). This may also be because of low statistical power.

The Stroke Council of the American Heart Association/American Stroke Association's updated definition of stroke broadly includes ischemic stroke, hemorrhagic stroke, and cerebral small vessel disease (CSVD, containing white matter hyperintensities (WMH)/silent brain infarcts (SBI) and cerebral microbleeds) [29]. Several of the studies that did not meet the inclusion criteria were cross-sectional studies, reporting relationships between Lp-PLA2 activity and CSVD risk. Performing a pooled RR for CVSD, we found that, per $1 \mathrm{SD}$ increase in Lp-PLA2 activity, there was an increased ischemic CSVD (WMH/SBI) risk (RR 1.31; 95\% CI 1.03-1.66; Appendix Supplement 8). Together, these results show that screening of Lp-PLA2 plasma levels may allow for the identification of patients at high risk of future cerebrovascular events, and particularly ischemic cerebrovascular events.

Although high Lp-PLA2 activity and mass were associated with increased risk of CHD and stroke, pharmacological lowering of Lp-PLA2 activity with darapladib does not significantly reduce cardiovascular (including stroke) events in patients who have experienced an ACS or with stable coronary heart disease $[11,30]$. Although two trials did not demonstrate clinical efficacy for darapladib, their limitations should be noted. Firstly, clinical efficacy may not be observed over 2.5 years [30] or a median of 3.7 years [11] in these studies. Secondly, the studies did not target a specific degree of Lp-PLA2 inhibition that has efficacy, although darapladib achieved an approximate reduction in Lp-PLA2 of $66 \%$ in the SOLID trial [30] and 65\% in the STABILITY trial [11]; in addition, many cardiovascular events that occur early after ACS or in stable CHD may be more associated with thrombotic mechanisms and may not be modifiable through Lp-PLA2 inhibition. These reasons may have limited the ability of these studies to discover a difference.

There are limitations to this study. We excluded studies that were unadjusted for confounding factors, which may have introduced bias. There was also a difference among 
included studies in the definitions of per SD change and highest compared with lowest Lp-PLA2 levels (eg. tertiles, quartiles, quintiles). Finally, the test methods for Lp-PLA2 were not uniform in the included studies, which is a potential source of bias. However, pooled RR in this meta-analysis did not change in the sensitivity analysis, suggesting that the pooled estimate is credible.

In conclusion, elevated Lp-PLA2 levels are associated with higher stroke risk. Lp-PLA2 levels can be considered as factor to predict stroke in high-risk individuals. Lp-PLA2 as a therapeutic target to prevent stroke requires further investigation.

\section{Data Availability}

The detailed data used to support the findings of this study are included within the article and the supplementary material files.

\section{Conflicts of Interest}

The authors report no financial or other conflicts of interest.

\section{Acknowledgments}

This study was supported by the Science and Technology Innovation Project from Foshan, Guangdong (FS0AA-KJ2181301-0006; FS0AA-KJ218-1301-0010).

\section{Supplementary Materials}

Appendix Supplement 1.PRISMA checklist. Appendix Supplement 2.Literature search strategy. Appendix Supplement 3.Quality assessment of the included studies. Appendix Supplement 4.Confounders adjusted in the included studies. Appendix Supplement 5.Assessment of publish bias of included studies by funnel plot and Egger's test in eligible studies. Appendix Supplement 6.Table S-1. Subgroup analyses of the association between Lp-PLA2 and risk of stroke. Appendix Supplement 7.Subgroup analyses based on per SD change of Lp-PLA2 activity. Appendix Supplement 8. Forest plot of Pooled RR and 95\%CI for CVSD with 1 SD higher LpPLA2 activity. (Supplementary Materials)

\section{References}

[1] G. A. Donnan, M. Fisher, M. Macleod, and S. M. Davis, "Stroke," The Lancet, vol. 371, no. 9624, pp. 1612-1623, 2008.

[2] K. Asano, S. Okamoto, K. Fukunaga et al., "Cellular source(s) of platelet-activating-factor acetylhydrolase activity in plasma," Biochemical and Biophysical Research Communications, vol. 261, no. 2, pp. 511-514, 1999.

[3] A. Zalewski and C. Macphee, "Role of lipoprotein-associated phospholipase A2 in atherosclerosis: Biology, epidemiology, and possible therapeutic target," Arteriosclerosis, Thrombosis, and Vascular Biology, vol. 25, no. 5, pp. 923-931, 2005.

[4] M. O'Donoghue, D. A. Morrow, M. S. Sabatine et al., "Lipoprotein-associated phospholipase A2 and its association with cardiovascular outcomes in patients with acute coronary syndromes in the PROVE IT-TIMI 22 (PRavastatin or atorVastatin evaluation and infection therapy-thrombolysis in myocardial infarction) trial," Circulation, vol. 113, no. 14, pp. 1745-1752, 2006.

[5] A. Thompson, P. Gao, L. Orfei et al., "Lipoprotein-associated phospholipase $\mathrm{A}_{2}$ and risk of coronary disease, stroke, and mortality: collaborative analysis of 32 prospective studies," The Lancet, vol. 375, pp. 1536-1544, 2010.

[6] D. Moher, A. Liberati, J. Tetzlaff, and D. G. Altman, "Preferred reporting items for systematic reviews and meta-analyses: the PRISMA statement," Annals of Internal Medicine, vol. 151, Article ID W64, pp. 264-269, 2009.

[7] G. A. Wells, B. Shea, D. O'Connell et al., "The Newcastle-Ottawa Scale (NOS) for assessing the quality if nonrandomized studies in meta-analyses," 2014, http://www.ohri.ca/programs/clinical_ epidemiology/oxford.asp.

[8] C. Wang, X. Fang, Y. Hua et al., "Lipoprotein-associated phospholipase A2 and risk of carotid atherosclerosis and cardiovascular events in community-based older adults in china," Angiology, vol. 69, no. 1, pp. 49-58, 2018.

[9] L. Han, C. Zhong, X. Bu et al., "Prognostic value of lipoproteinassociated phospholipase A2 mass for all-cause mortality and vascular events within one year after acute ischemic stroke," Atherosclerosis, vol. 266, pp. 1-7, 2017.

[10] P. Ganz, P. Amarenco, L. B. Goldstein et al., "Association of osteopontin, neopterin, and myeloperoxidase with stroke risk in patients with prior stroke or transient ischemic attacks," Stroke, vol. 48, pp. 3223-3231, 2017.

[11] L. Wallentin, C. Held, P. W. Armstrong et al., "Lipoproteinassociated phospholipase A2 activity is a marker of risk but not a useful target for treatment in patients with stable coronary heart disease," Journal of the American Heart Association, vol. 5, 2016.

[12] A. Shoamanesh, S. R. Preis, A. S. Beiser et al., "Circulating biomarkers and incident ischemic stroke in the framingham offspring study," Neurology, vol. 87, no. 12, pp. 1206-1211, 2016.

[13] Y. Pokharel, W. Sun, L. M. Polfus et al., "Lipoprotein associated phospholipase A2 activity, apolipoprotein C3 loss-of-function variants and cardiovascular disease: the atherosclerosis risk in communities study," Atherosclerosis, vol. 241, no. 2, pp. 641-648, 2015.

[14] J. Lin, H. Zheng, B. L. Cucchiara et al., "Association of Lp-PLA 2-A and early recurrence of vascular events after TIA and minor stroke," Neurology, vol. 85, no. 18, pp. 1585-1591, 2015.

[15] M. Katan, Y. P. Moon, M. C. Paik, R. L. Wolfert, R. L. Sacco, and M. S. V. Elkind, "Lipoprotein-associated phospholipase A2 is associated with atherosclerotic stroke risk: the northern manhattan study," PLoS ONE, vol. 9, no. 1, Article ID e83393, 2014.

[16] N. R. Cook, N. P. Paynter, J. E. Manson et al., "Clinical utility of lipoprotein-associated phospholipase A2 for cardiovascular disease prediction in a multiethnic cohort of women," Clinical Chemistry, vol. 58, no. 9, pp. 1352-1363, 2012.

[17] M. J. Caslake, C. J. Packard, M. Robertson et al., "Lipoproteinassociated phospholipase A2, inflammatory biomarkers, and risk of cardiovascular disease in the prospective study of pravastatin in the elderly at risk (PROSPER)," Atherosclerosis, vol. 210, no. 1, pp. 28-34, 2010.

[18] N. S. Jenny, C. Solomon, M. Cushman et al., "Lipoproteinassociated phospholipase A2 (Lp-PLA2) and risk of cardiovascular disease in older adults: results from the cardiovascular health study," Atherosclerosis, vol. 209, no. 2, pp. 528-532, 2010. 
[19] HPSGroup, "Lipoprotein-associated phospholipase A(2) activity and mass in relation to vascular disease and nonvascular mortality," Journal of Internal Medicine, vol. 268, no. 4, pp. 348358, 2010.

[20] S. Tsimikas, J. Willeit, M. Knoflach et al., "Lipoproteinassociated phospholipase A2 activity, ferritin levels, metabolic syndrome, and 10-year cardiovascular and non-cardiovascular mortality: results from the bruneck study," European Heart Journal, vol. 30, no. 1, pp. 107-115, 2009.

[21] M. S. V. Elkind, W. Tai, K. Coates, M. C. Paik, and R. L. Sacco, "Lipoprotein-associated phospholipase $\mathrm{A}_{2}$ activity and risk of recurrent stroke," Cerebrovascular Disease, vol. 27, no. 1, pp. 4250, 2009.

[22] S. J. Robins, D. Collins, J. J. Nelson, H. E. Bloomfield, and B. F. Asztalos, "Cardiovascular events with increased lipoprotein-associated phospholipase $\mathrm{A}(2)$ and low highdensity lipoprotein-cholesterol: the veterans affairs hdl intervention trial," Arteriosclerosis, Thrombosis, and Vascular Biology, vol. 28, no. 6, pp. 1172-1178, 2008.

[23] M. Persson, G. Berglund, J. J. Nelson, and B. Hedblad, "Lp-PLA2 activity and mass are associated with increased incidence of ischemic stroke: a population-based cohort study from Malmo, Sweden," Atherosclerosis, vol. 200, no. 1, pp. 191-198, 2008.

[24] M. S. Sabatine, D. A. Morrow, M. O’Donoghue et al., "Prognostic utility of lipoprotein-associated phospholipase A2 for cardiovascular outcomes in patients with stable coronary artery disease," Arteriosclerosis, Thrombosis, and Vascular Biology, vol. 27, no. 11, pp. 2463-2469, 2007.

[25] M. S. V. Elkind, W. Tai, K. Coates, M. C. Paik, and R. L. Sacco, "High-sensitivity C-reactive protein, lipoproteinassociated phospholipase A2, and outcome after ischemic stroke," JAMA Internal Medicine, vol. 166, no. 19, pp. 2073-2080, 2006.

[26] C. M. Ballantyne, R. C. Hoogeveen, H. Bang et al., "Lipoproteinassociated phospholipase A2, high-sensitivity C-reactive protein, and risk for incident ischemic stroke in middle-aged men and women in the atherosclerosis risk in communities (ARIC) study," JAMA Internal Medicine, vol. 165, no. 21, pp. 2479-2484, 2005.

[27] H. H. Oei, I. M. van der Meer, A. Hofman et al., "Lipoproteinassociated phospholipase A2 activity is associated with risk of coronary heart disease and ischemic stroke: the rotterdam study," Circulation, vol. 111, no. 5, pp. 570-575, 2005.

[28] A. De Stefano, L. Mannucci, F. Tamburi et al., "Lp-PLA2, a new biomarker of vascular disorders in metabolic diseases," International Journal of Immunopathology and Pharmacology, vol. 33, Article ID 205873841982715, 2019.

[29] R. L. Sacco, S. E. Kasner, J. P. Broderick et al., "An updated definition of stroke for the 21st century: a statement for healthcare professionals from the American heart association/american stroke association," Stroke, vol. 44, no. 7, pp. 2064-2089, 2013.

[30] M. L. O'Donoghue, E. Braunwald, H. D. White et al., "Effect of darapladib on major coronary events after an acute coronary syndrome: the SOLID-TIMI 52 randomized clinical trial," Journal of the American Medical Association, vol. 312, no. 10, pp. 1006-1015, 2014. 


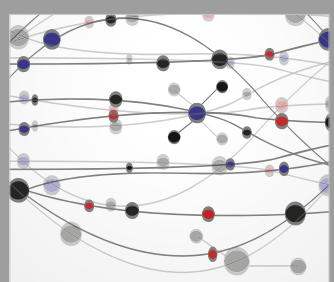

The Scientific World Journal
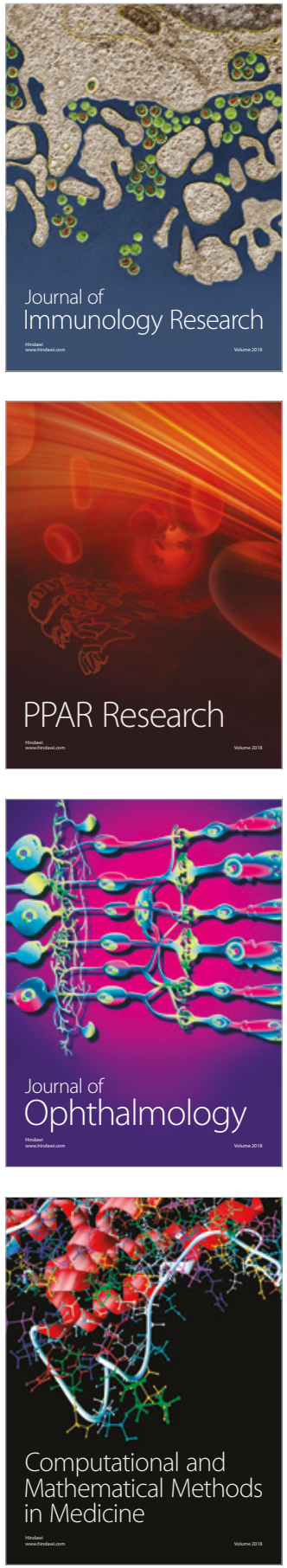

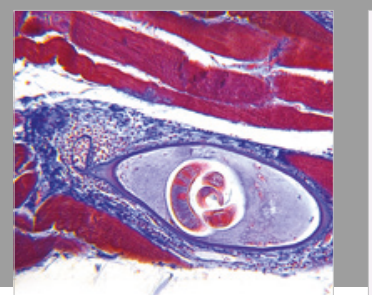

Gastroenterology Research and Practice

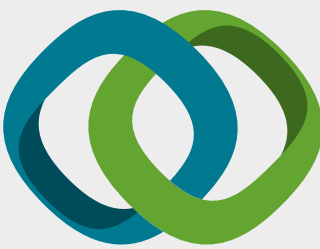

\section{Hindawi}

Submit your manuscripts at

www.hindawi.com
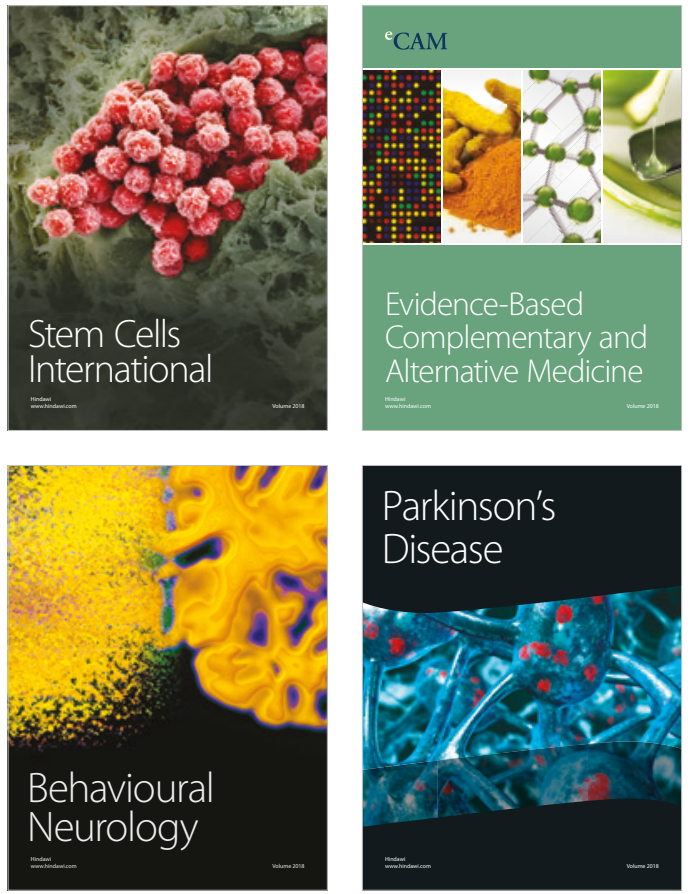

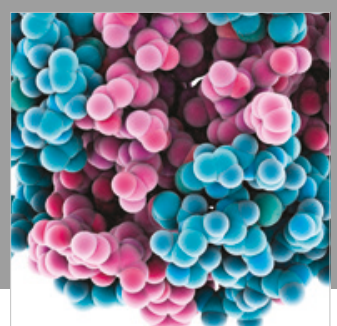

ournal of

Diabetes Research

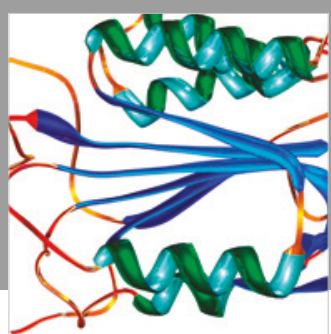

Disease Markers
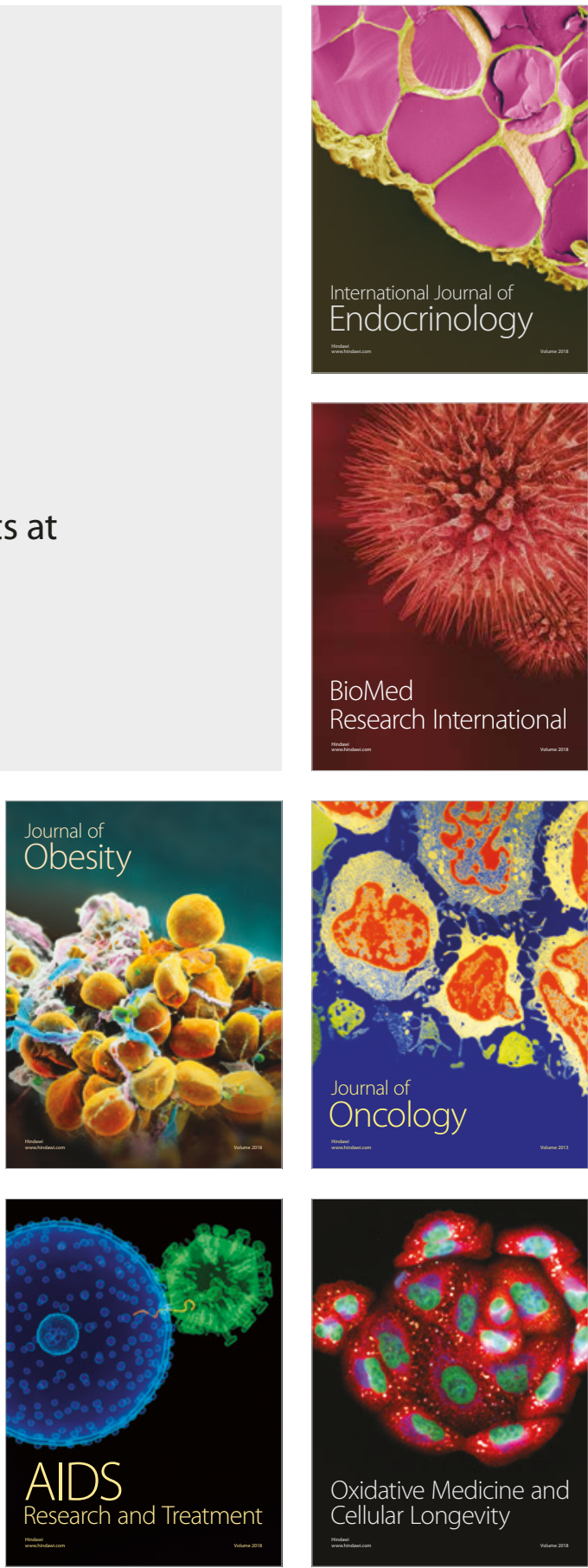